\title{
Phase based 3D Texture Features
}

\author{
Janis Fehr ${ }^{1}$ and Hans Burkhardt ${ }^{1}$ \\ Albert-Ludwigs-Universität Freiburg, Institut für Informatik, Lehrstuhl für \\ Mustererkennung und Bildverarbeitung, Georges-Koehler-Allee Geb. 052, \\ 79110 Freiburg, Deutschland \\ fehr@informatik.uni-freiburg.de, \\ http://lmb.informatik.uni-freiburg.de/
}

\begin{abstract}
In this paper, we present a novel method for the voxel-wise extraction of rotation and gray-scale invariant features. These features are used for simultaneous segmentation and classification of anisotropic textured objects in 3D volume data. The proposed new class of phase based voxel-wise features achieves two major properties which can not be achieved by the previously known Haar-Integral based gray-scale features [1]: invariance towards non-linear gray-scale changes and a easy to handle data driven feature selection. In addition, the phase based features are specialized to encode 3D textures, while texture and shape information interfere in the Haar-Integral approach. Analog to the HaarIntegral features, the phase based approach uses convolution methods in the spherical harmonic domain in order to achieve a fast feature extraction.

The proposed features were evaluated and compared to existing methods on a database of volumetric data sets containing cell nuclei recorded in tissue by use of a 3D laser scanning microscope.
\end{abstract}

\section{Introduction}

Segmentation and classification of anisotropic objects in 3D volume data, especially of biological structures in 3D laser scanning microscope (LSM) images, has recently become a fast rising topic. Life sciences take more and more advantage of 3D imaging techniques like LSM, combined with fluorescent antibody markers or auto-fluorescent probes. For a broad band of research topics from cellular anatomy to gene expression experiments, 3D volumetric imaging methods are used. Microscopes of the latest generation allow very fast, high resolution, multi-channel recordings which produce high amounts of data. At this stage, there is a rising demand for (semi)automatic image analysis methods which on one hand would allow high-throughput experiments, and on the other hand, provide a tool for quantitative data analysis. Most of the demanded automatic analysis tasks include the "basic" but difficult operations of segmentation, classification, or landmark detection for registration of textured objects in 3D volume data. All these operations have in common, that a rotational invariant representation of the $3 \mathrm{D}$ data is needed.

This paper is structured as follows: first we give a brief overview of related work, 
especially the Haar-Integral based gray-scale features are revised. Then we motivate the phase based approach. In section 2 the proposed features are discussed in detail. Section 3 introduces the data driven selection of phase based features. Experiments are presented in section 4 .

\section{$1.1 \quad$ Related Work}

To our best knowledge, there are not many publications available regarding the segmentation or classification of anisotropic 3D objects in LSM volume data. [2] presented a method for automatic segmentation of cell nuclei in dilution using region-growing (watershed) techniques. However, this method fails for recordings of nuclei in dense tissue probes as we present in our experiments. In general, we found that standard edge based, contour based, or region-growing segmentation methods deliver poor results on tissue probes. This is due the fact, that difficult segmentation tasks require a great amount of a-priori knowledge, which can not be encoded in these methods. Model driven segmentation methods, such as snakes or level-sets, are capable to encode a-priori knowledge and therefore might work well on single examples of this data. But, since there is a high variability in biological structures like cell nuclei and the demand is going towards very general and easy adaptable methods, the use of such models is complex and not flexible enough for many biological tasks.

Learning Segmantion by Example: An other way of incorporating apriori knowledge into a segmentation task was presented in [3]. Here segmentation and classification is performed voxel-wise and in a single step using training examples given by a human expert. In an iterative process, the expert labels some voxels of different object classes and background. Then voxel-wise invariant features are extracted and each voxel is classified based on the given training samples. Neighboring voxels of the same label are then grouped to objects. The resulting segmentation/classification is adjusted by the expert until the model reaches a stable state. Afterwards new datasets can be segmented using this model. Models can be adopted to new cell types or even totally new data by retraining the model with additional training samples. We use this method combined with a Support Vector Machine (SVM) [4][5] classifier and our proposed phase based features for the experiments in section 4 .

Voxel-Wise Haar-Integration Features: [1] and [6] introduced voxelwise rotation invariant gray-scale features for combination with the previously described simultaneous segmentation and classification algorithm. In general, (rotation) invariance can be achieved via integration over the transformation (rotation) group:

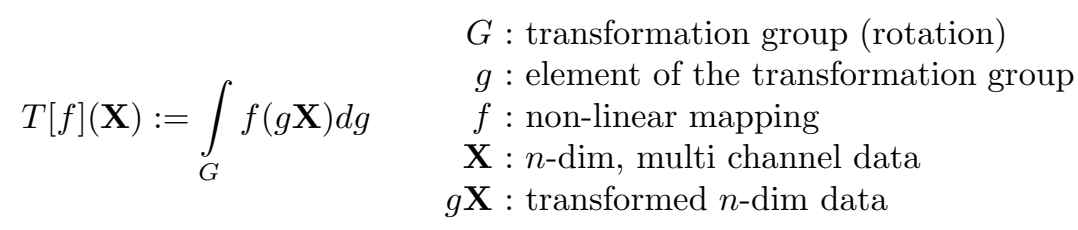


[1] and [6] formulated this approach for the special case of 3D "2-point" and "3-point" gray-scale invariants of the form (here given for "3-point"):

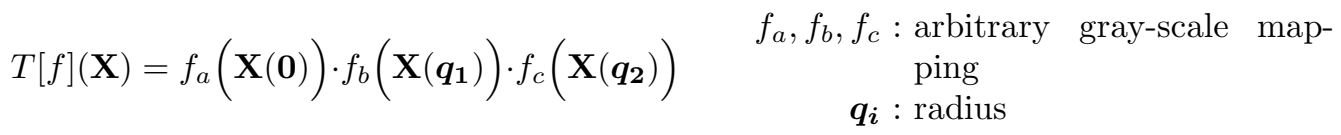

and showed a fast way of voxel-wise calculation via convolution in the spher-
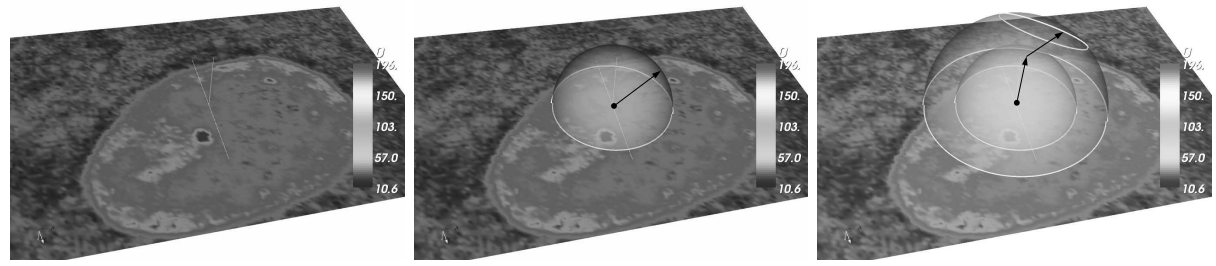

Fig. 1. Schematic calculation of Haar-Integral features. Left: slice through cell nuclei in original data. Center: calculation of "2-Point" invariants via Haar-Integration along the surface of a sphere. Right: "3-Point" features incorporate the relationship of gray-values at three points (center and two on concentric spheres). In this case many degrees of freedom have to be covered in order to achieve rotational invariance.

ical harmonic domain. Fig. (1) illustrates the calculation of gray-scale HaarIntegration features.

The results which can be achieved with this method are very reasonable (as shown in [1]), but the Haar-Integration approach also has some drawbacks: first, the features are not invariant towards gray-scale shifts, which appear in recordings moving deeper into the specimen. Due to the integral nature, the mean gray-value tends to dominate the value of the invariants and only a gray-scale robustness can be achieved via elaborate normalization techniques. Second, the Haar-Features have many degrees of freedom $\left(\boldsymbol{q}_{\mathbf{1}}, \boldsymbol{q}_{\mathbf{2}}, f_{a}, f_{b}, f_{c}\right)$, which makes an extensive feature selection necessary. And last but not least, the integration step makes it almost impossible to conduct an inverse inference from discriminating features to the original structure, which would be very useful for a deeper understanding and further improvements of the method.

\section{Phase based 3D Texture Features}

In order to overcome the drawbacks of the Haar-Features while utilizing its strengths, we propose a new phase based approach towards voxel-wise rotation and gray-scale invariant features. As for the Haar-Features, we encode the spherical neighborhood of a voxel to an invariant feature vector. Since this feature calculation is conducted in the spherical harmonic domain, we first give a brief introduction to the harmonic methods used for our approach. 


\subsection{Spherical Harmonics}

To represent the neighborhood of some point in a 3D Euclidean space as a function $f$ on the surface of a sphere (parameterized over the two angles $\theta$ and $\phi$ ), the original 3D signal can be expanded in terms of spherical harmonics [7]. These provide an orthogonal basis for such functions analog to the Fourier transform in Euclidean space. This way, every spherical function can be represented by the sum of its harmonics:

$$
f(\theta, \phi)=\sum_{l=0}^{\infty} \sum_{m=0}^{l} a_{l m} Y_{l}^{m}(\theta, \phi)
$$

where $l$ denotes the band of expansion, $m$ the number of components for the $l$-th band and $a_{l m}$ the harmonic coefficient. The harmonic base functions $Y_{l}^{m}(\theta, \phi)$ are calculated as follows:

$$
Y_{l}^{m}(\theta, \phi)=\sqrt{\frac{2 l+1}{4 \pi} \frac{(l-m) !}{(l+m) !}} \cdot P_{l}^{m}(\cos \theta) e^{i m \phi}
$$

with the associated Legendre polynomial $P_{l}^{m}$.

Note that in this formulation we take advantage of the symmetry in the harmonic representation, neglecting the negative coefficients. For practical reasons we also split the base components into their real and imaginary parts following the notation $Y_{l}^{m r}$ and $Y_{l}^{m c}$ respectively. Fig. (2) shows the first few spherical harmonics.

The transformation $D \widehat{(l, m)}$ of the original volumetric data $D$ into the harmonic domain is easily computed via fast convolution:

$$
\widehat{D(l, m})=Y_{l}^{m}(\theta, \phi) * D
$$

where $*$ denotes a convolution in Euclidean space and $Y_{l}^{m}(\theta, \phi)$ a spherical harmonic base component.

\subsection{Feature Calculation}

In order to obtain local features $\left.D_{r} \widehat{(l, m}\right)$ which encode the spherical neighborhood of each voxel at different consecutive radii $r$, we restrict the harmonic expansion to the surface of spheres $S_{r}$ smoothed by a Gaussian filter $\mathcal{G}$.

$$
\left.D_{r} \widehat{(l, m}\right)=\left(Y_{l}^{m}(\theta, \phi) \cdot\left(S_{r} * \mathcal{G}\right)\right) * D
$$

Once the original volume data is transformed to the harmonic domain, there are different ways of calculating a rotational invariant representation. The simplest and well known approach is to take the band-wise absolute value of the harmonic coefficients, also known as harmonic descriptors, which for example have been used in [8] for 3D object recognition. However, this method yields a major drawback: by taking the absolute values one totally neglects the the relations between 
1st Band 2nd Band 3rd Band 4th Band 5th Band

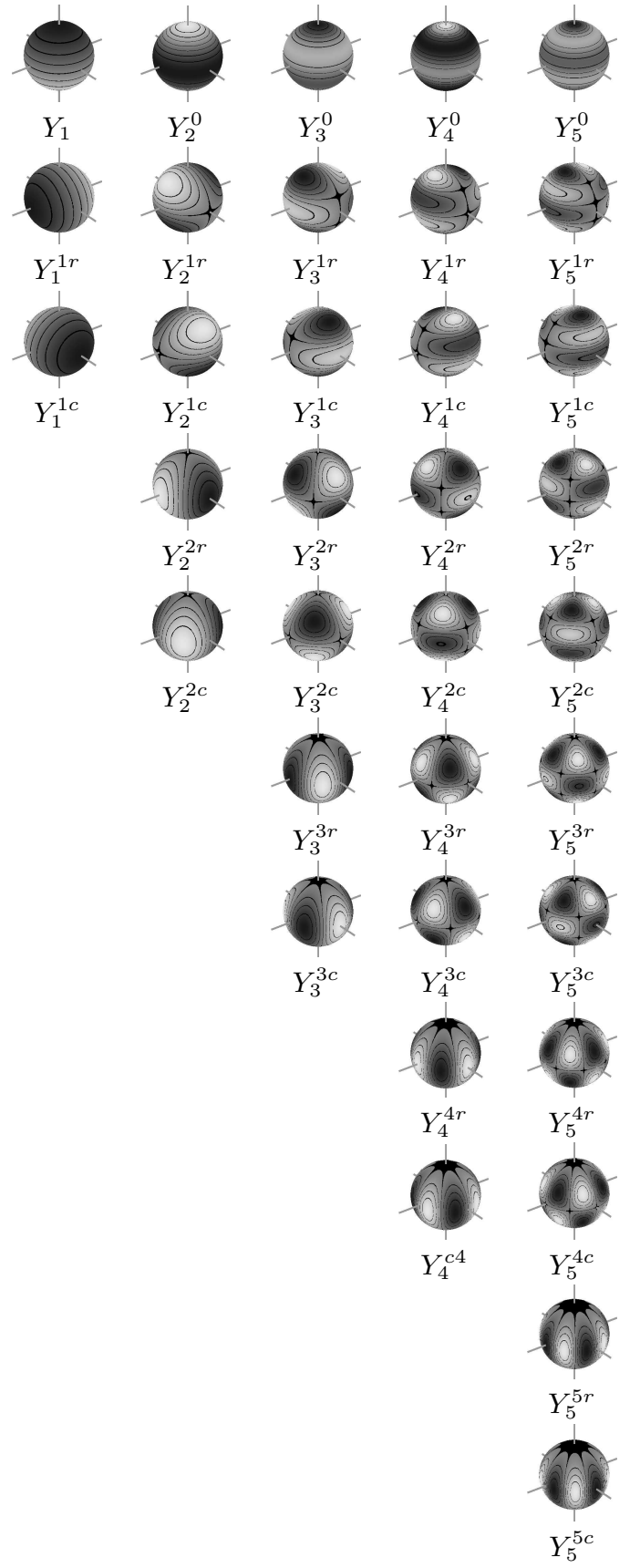

Fig. 2. Spherical harmonics on a sphere surface from 1st to 5th band. 
the bands of the harmonic representation. This leads to ambiguous features with decreased separability.

For the Haar-Integration features the rotation invariance is achieved by integration over all possible rotations (in terms of convolutions in the harmonic domain). Here the band relations are implicitly conserved, but as mentioned before, the integration inhibits a gray-scale invariance. As the name "phase-based" features suggests, our new method uses only the relation of the harmonic bands as feature representation. This approach is motivated by results known from Fourier transform, which showed that the characteristic information is dominant in the phase of a signal's spectrum rather than in the pure magnitude of it's coefficients. Following this strategy has the nice side-effect that the overall gray-value intensity is only encoded in the amplitude, making a phase-only method directly gray-scale invariant.

Phase: In this paper, the phase of a local harmonic expansion in band $l$ a radius $r$ is denoted by the orientation of the vector $\mathbf{P}_{\mathbf{l}, \mathbf{r}}$, containing the $l$ harmonic coefficient components of the band-wise local expansion (Equ. 6). Since the coefficients are changing when the underlying data is rotated, the phase itself is not a rotational invariant feature.

$$
\mathbf{P}_{\mathbf{1}, \mathbf{r}}(m):=D_{r} \widehat{(l, m)} /\left\|\widehat{D_{r}}\right\|
$$

Due to the orthogonality of the harmonic base, the rotation of a spherical neighborhood can be calculated in the harmonic domain via a band-wise matrix multiplication of a symmetric and orthogonal rotation matrix $\mathbf{R}_{l}$ of size $l \times l$ with the harmonic coefficients in the $l$-th band. Unfortunately it turns out, that the actual calculation of this rotation matrix is getting more and more complicated and time consuming for higher bands. This would make it very expensive to achieve rotation invariance of the phase via pre-alignment.

But, there is another way to realize rotational invariant phase-only features: since we are interested in encoding the neighborhood at many consecutive radii, we can take advantage of this additional information and construct a phase-only rotational invariant feature based on the band-wise relations of phases between the different concentric harmonic series.

Fig. (3) illustrates the basic idea: the relation (angle) between phases of harmonic expansions at different radii, but in the same harmonic band, are invariant towards rotation around the center of the expansion. Intuitively phases in the same harmonic band undergo the same changes under rotation of the underlying data, keeping the angle between the phases of different radii constant. We encode this angle in terms of the dot product of band-wise spherical harmonic expansions. The resulting phase-only features can be interpreted as a description of the change in the 3D data texture, when moving from one spherical neighborhood to the next concentric neighborhood. We use this texture encoding property in the next section to find discriminatory texture elements for classes of 3D anisotropic volumetric objects. The formalization of the band-wise phase based feature vector $T\left[f_{l}\right]$ calculation is given as the dot product between two 


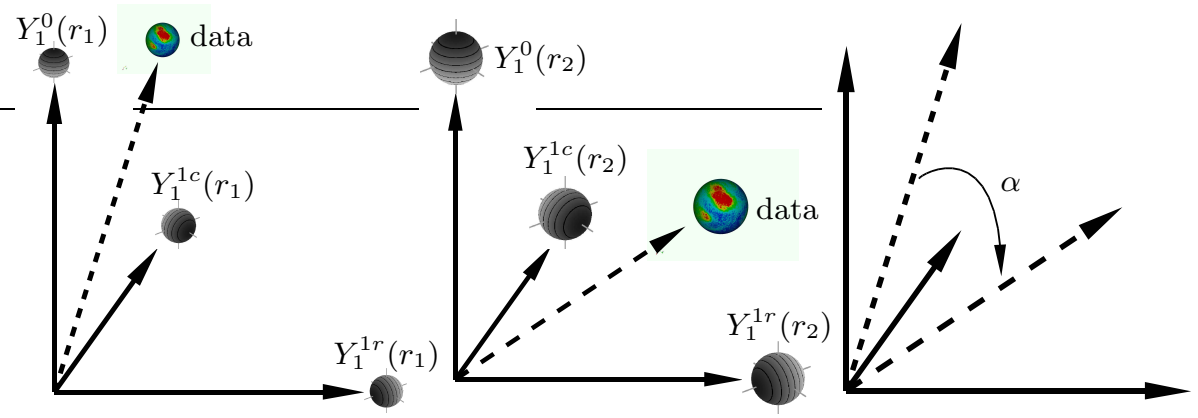

Fig. 3. Schematic example of the phase based feature calculation. Left: representation of the original data as combination of the 3D base functions of an expansion in the 1st band at radius $r_{1}$. Center: representation at radius $r_{2}$. Right: the feature is encoding the 1 st band phase angle $\alpha$ between the two concentric harmonic expansions.

band-wise expansions at radii $r_{1}$ and $r_{2}$ :

$$
T\left[f_{l}\right]:=<P_{l_{r 1}}, P_{l_{r 2}}>
$$

Proof of rotational invariance is rather straight forward basic linear algebra: Since the phases of both radii are in the same band, a rotation of the underlying data can now be expressed in terms of matrix multiplications with the same orthogonal rotation matrix $\mathbf{R}_{l}$ :

$$
\begin{array}{rlr}
T^{\prime}\left[f_{l}\right] & =<\mathbf{R}_{l} P_{l_{r 1}}, \mathbf{R}_{l} P_{l_{r 2}}> & \\
& =\left(\mathbf{R}_{l} P_{l_{r 1}}\right)^{T}\left(\mathbf{R}_{l} P_{l_{r 2}}\right) & \text { rewrite as matrix multiplication } \\
& =\left(P_{l_{r 1}}\right)^{T}\left(\mathbf{R}_{l}\right)^{T}\left(\mathbf{R}_{l} P_{l_{r 2}}\right) & \text { resove transposition } \\
& =\left(P_{l_{r 1}}\right)^{T}\left(\mathbf{R}_{l}^{T} \mathbf{R}_{l}\right)\left(P_{l_{r 2}}\right) & \text { comutativity } \\
& =\left(P_{l_{r 1}}\right)^{T} \underbrace{\left(\mathbf{R}_{l}^{T} \mathbf{R}_{l}\right)}_{=I}\left(P_{l_{r 2}}\right) & \text { use orthogonality of } \mathbf{R}_{l} \\
& =\left(P_{l_{r 1}}^{T} P_{l_{r 2}}\right) \\
& =<P_{l_{r 1}}, P_{l_{r 2}}> \\
& =T\left[f_{l}\right]
\end{array}
$$

Since the rotational invariance is achieved band wise, the approximation of the original data via harmonic expansion can be cut off at an arbitrary band, encoding just the level of detail needed for the application.

Computational complexity: compared to the Haar-Features the calculation of a single phase based feature has about the same computational complexity, both include expansions of two different neighborhoods in harmonics and their dot-product. However, a significant speedup can be achieved by the usage of phase based features. Our experiments showed, that due to the higher discrimination cability, the number of needed features is significant lower compared to the usage of Haar-Features. 


\section{Data driven Features}

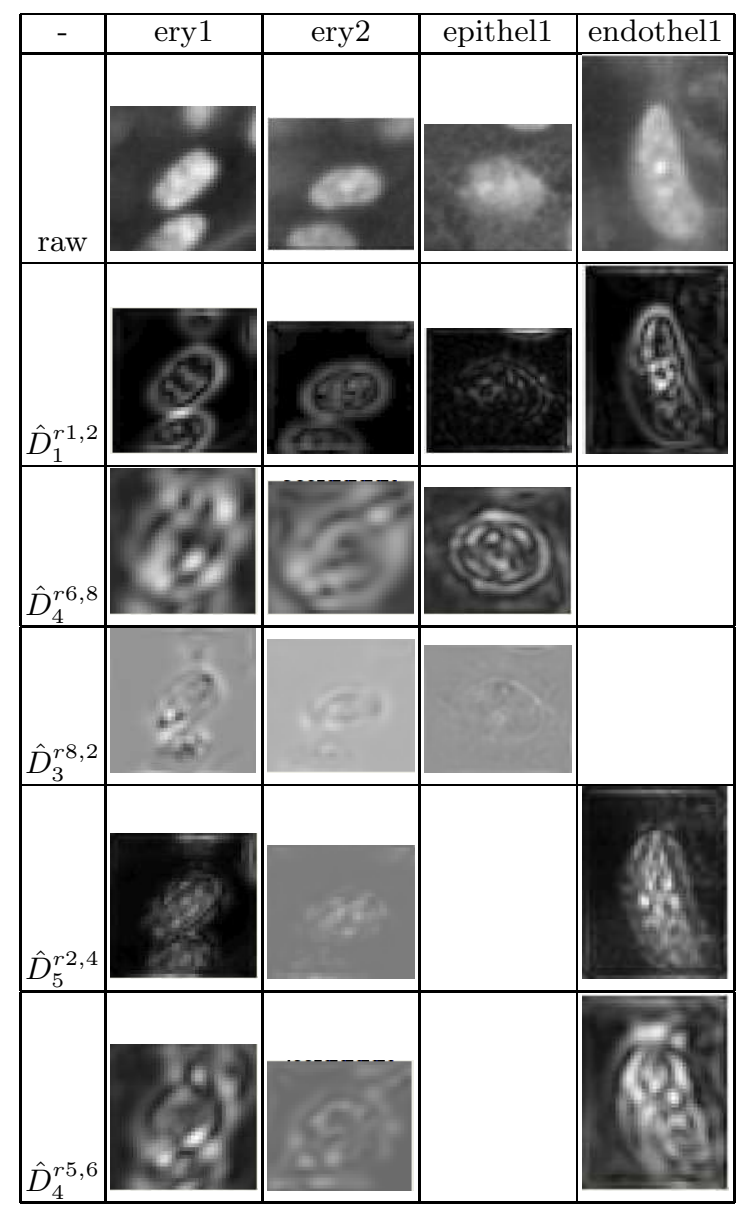

Fig. 4. Distinguishing feature response of different cell nuclei. $\hat{D}_{1}^{r 1,2}$ denotes a feature in band 1 encoding the phase change from radius 1 to 2 .

As Fig. (4) shows an example of how different 3D textures (of nuclei types) lead to distinguishable representations in feature space. Fine, high frequency textures have an large impact on features in higher bands and at small radii while a more raw textured object is predominantly represented in the lower bands and larger radii. In order to determine the most distinguishable features, we apply a strong gauss filter to the features. This way the very local but strong texture responses are distributed to the local neighborhood. Then we apply the maximum marginal diversity algorithm [9] to calculate to most separating features. Since the phase based features are independent for every band, the data representation can be 
reduced to arbitrary band and radii combinations without a full transformation to the harmonic domain.

\section{Experiments}

To verify our new method, we conducted some first experiments on a database of 3D laser-scanning microscope (LSM) recordings from cell nuclei in tissue.

Data: The database consists of 236 nuclei samples divided in 5 different classes (erythrocyte, endothelia cells, pericyte, fibroblast and macrophage). The samples were recored from tissue probes of the chicken chorioallantoic membrane which were treated as described in [10]. Human experts manually segmented and labeled the sample nuclei recordings as ground truth.

Methods: We extracted 16 features involving 8 different radii and expan-
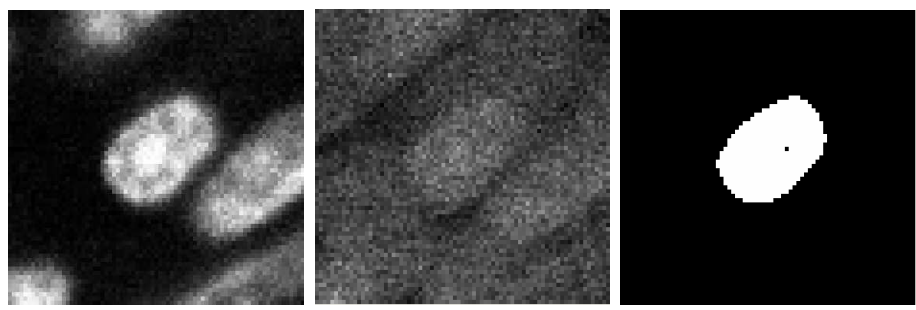

Fig. 5. Slice of a sample 3D database entry (erythrocyte). Left: YoPro stained channel. Center: SNAAlexa stained channel. Right: ground truth segmentation and label.

sions up to the 6th band. All features were selected from a larger number of initial features by the data driven selection method. Two reference models were trained on two small disjunct subsets of the database, containing samples from two different recording depths. The different depths cause shifts in the gray values of the recordings, as described in section 1 . The remaining samples were also split into two sets, according to the recording depths, and were then classified with both models.

Results: We compared non gray-scale invariant 3-point Haar-Integral (gsi) features (as described in [3]) and the new, gray-scale invariant, phase based features. While the gsi features performed very well for constant gray-scales (94.53 $\%$ ), the classification rate dropped to poor $46.2 \%$ for the subsets from a different recording depth. The phase based features on the other hand performed slightly worse on constant gray-scales $(91,58 \%)$, but delivered stable results for varying gray values: $90.1 \%$.

\section{Conclusion and Outlook}

In this paper we presented a novel approach of calculating rotational and grayscale invariant 3D texture features based on the phase information of a spherical 
harmonic expansion of the original data. Our first experiments showed promising results and pointed out the strengths of these new features, especially concerning gray-scale changes in 3D textures as well as the possibility to construct data driven features in comparison to Haar-Integration features.

For future work, we will continue to focus on methods for data driven features. Seeking for ways of learning the most discriminative features for larger local areas, moving towards a 3D patch based approach.

\section{References}

1. Ronneberger, O., Fehr, J., Burkhardt, H.: Voxel-wise gray scale invariants for simultaneous segmentation and classification. In Proceedings of the 27th DAGM Symposium, in number 3663 LNCS, Springer, Vienna, Austria, 30.8 - 2.9. 2005. (2005)

2. Wählby, C., e.a.: Compining intensity, edge, and shape information for $2 \mathrm{~d}$ and $3 \mathrm{~d}$ segmentation of cell nuclei in tissue sections. (Journal of Microscopy, July 2004, vol. 215, no. 1, pp. 67-76(10))

3. Fehr, J., Ronneberger, O., Kurz, H., Burkhardt, H.: Self-learning segmentation and classification of cell-nuclei in $3 \mathrm{~d}$ volumetric data using voxel-wise gray scale invariants. Proceedings of the 27th DAGM Symposium, in number 3663 LNCS, Springer, Vienna, Austria, 30.8 - 2.9. 2005. (2005)

4. Ronneberger, O.: Libsvmtl - a support vector machine template library. download at: http://lmb.informatik.uni-freiburg.de/lmbsoft/libsvmtl/ (2004)

5. Vapnik, V.N.: The nature of statistical learning theory. Springer (1995)

6. Ronneberger, O., Fehr, J., Burkhardt, H.: Voxel-wise gray scale invariants for simultaneous segmentation and classification - theory and application to cell-nuclei in 3d volumetric data. Internal report 2/05, IIF-LMB, University Freiburg (2005)

7. Groemer, H.: Geometric Applications of Fourier Series and Spherical Harmonics. Cambridge University Press (1996)

8. M. Kazhdan, T.F., Rusinkiewicz, S.: Rotation invariant spherical harmonic representation of 3d shape descriptors. in Symposium on Geometry Processing (2003)

9. Vasconecelos, N.: Feature selection by maximum marginal diversity: optimality and implications for visual recognition. Proceedings of IEEE Conf. on Computer Vision and Pattern Recogniton, Madison, USA (2003)

10. Kurz, H., Papoutsi, M., Wilting, J., Christ, B.: Pericytes in experimental mdamb231 tumor angiogenesis. Histochem Cell Biol (2002) 117:527-534 